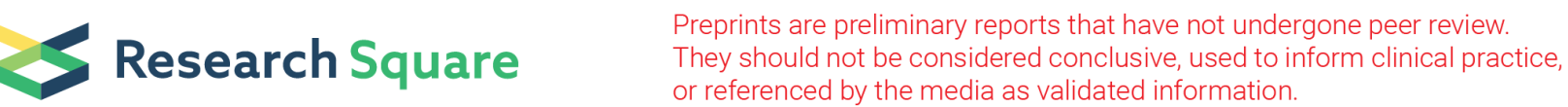

\section{Comparative Risk of Incidence and Clinical Outcomes of COVID-19 among Proton Pump Inhibitor and Histamine-2 Receptor Antagonist Users: A Nationwide Retrospective Cohort Study}

Jimyung Park

Ajou University Graduate School of Medicine

Seng Chan You

Yonsei University College of Medicine

Jaehyeong Cho

Ajou University Graduate School of Medicine

Chan Hyuk Park

Hanyang University Guri Hospital, Hanyang University College of Medicine

Woon Shin

Hallym University College of Medicine

Rae Woong Park

Ajou University Graduate School of Medicine

Seung In Seo ( $\square$ doctorssi@kdh.or.kr)

Hallym University College of Medicine

Research Article

Keywords: Retrospective Studies, Pharmacovigilance, Epidemiology, Acid Suppressants

Posted Date: October 5th, 2021

DOI: https://doi.org/10.21203/rs.3.rs-882550/v1

License: (c) (1) This work is licensed under a Creative Commons Attribution 4.0 International License.

Read Full License 


\section{Abstract}

Background: This study aimed to evaluate incidence risk and severe clinical outcomes in COVID-19 disease among short-term users of acid-suppressants in South Korea.

Methods: This retrospective cohort study, conducted using a nationwide claims database for South Korea, used data from patients with COVID-19 tested between January 1 and May 15, 2020. Patients aged over 18 years and prescribed proton pump inhibitors (PPI) or histamine-2 receptor antagonist $\left(\mathrm{H}_{2} \mathrm{RA}\right)$ for more than 7 days were identified. Primary outcome was COVID-19 while secondary outcomes were all-cause mortality, hospitalization with respiratory disease, or intensive respiratory intervention. Large-scale propensity scores were used to match patients, while the Cox proportional hazard model was utilized to evaluate any association between exposure and outcome(s). The risk estimates were calibrated by using 123 falsification endpoints.

Results: We identified 26,166 PPI users and $62,117 \mathrm{H}_{2}$ RA users. After propensity score matching, compared to $\mathrm{H}_{2}$ RA use, PPI use was not significantly associated with lower risk of COVID-19 (calibrated hazard ratio [HR], 0.81 [95\% confidence interval ( $\mathrm{Cl}), 0.30-2.19]$ ); moreover, PPI use was not associated with adverse clinical outcomes in COVID-19, namely, hospitalization with respiratory disease (calibrated $\mathrm{HR}, 0.88$ [95\% Cl, 0.72-1.08]), intensive respiratory interventions (calibrated $\mathrm{HR}, 0.92$ [95\% Cl, 0.46-1.82]), except for all-cause mortality (calibrated HR, 0.54 [95\% Cl, 0.31-0.95]).

Conclusions: In this study, we found that the PPI user was not associated with risk of COVID-19 compared to $\mathrm{H}_{2} \mathrm{RA}$ users. There was no significant relationship between severe clinical outcomes of COVID-19 and exposure to PPI compared with $\mathrm{H}_{2} \mathrm{RA}$, except for all-cause mortality.

\section{Introduction}

Proton pump inhibitors (PPI) are the mainstay in the management of acid-related gastrointestinal disease, including gastroesophageal reflux disease and peptic ulcer disease, and for the prevention of $\mathrm{GI}$ bleeding and stress ulcers. ${ }^{1}$ While their widespread use has resulted in the improvement of acid-related disorders, concerns about potential complications due to PPI use, such as osteoporosis, dementia, malabsorption, gastrointestinal neoplasia, and increased susceptibility to bacterial infection, have also been rising. ${ }^{2,3}$ Further, it is possible that acid-suppressant drugs could increase susceptibility to respiratory infections because they counter the acidic environment of stomach, thereby allowing bacterial colonization. ${ }^{45}$ Even though several studies have evaluated the association between pneumonia and acid-suppressive drugs, the results remain inconclusive. ${ }^{4,6-10}$

Recently, several studies have described the effects of acid-suppressive agent use on the clinical course of and susceptibility to SARS-CoV-2 infection (COVID-19) ${ }^{11-15}$ however, few studies have directly compared incidence and risk of complications in COVID-19 between PPI and histamine-2 receptor antagonist $\left(\mathrm{H}_{2} \mathrm{RA}\right)$ therapy. In 2011, a systematic review and meta-analysis by Eom et al. investigated the 
association between use of acid-suppressive drugs and risk of pneumonia and found that the overall risk was higher among people using PPIs than $\mathrm{H}_{2}$ RAs (adjusted odds ratio [OR] 1.27, 95\% confidence interval [Cl] $1.11-1.46$ vs. $1.22,95 \% \mathrm{Cl} 1.09-1.36)^{7}$. Nonetheless, the influence of acid-suppressive drugs on the viral pneumonia remains controversial.

To date, only limited data is available on the relationship between acid suppression therapy and clinical course of COVID-19 infection; therefore, we conducted a population-based retrospective cohort study to compare the risk of complications in COVID-19 among Korean patients prescribed PPI and $\mathrm{H}_{2} \mathrm{RA}$ therapy.

\section{Methods}

\section{Data Sources}

A national claims database for South Korea that included data on COVID-19 testing was obtained from the Health Insurance Review and Assessment service (HIRA) and used in this study. ${ }^{16}$ HIRA is the South Korean national institution for reviewing and assessing national health insurance claims. In response to the COVID-19 pandemic, HIRA collected data on COVID-19 testing by the reverse transcriptase polymerase chain reaction method from 1 January to 15 May, 2020. Notably, the collected data were converted into the Observational Medical Outcomes Partnership (OMOP) common data model (CDM), version 5, and released to the public. Hospitalization records were extracted for all patients involved in the study. This study was approved by the institutional review board of the Kangdong Sacred Heart Hospital (no. 202004-001).

\section{Study population and exposure}

We identified adult patients aged 18 years or above who had not been diagnosed with COVID-19 disease. The cohort comprised patients who had been prescribed acid-suppressants and had taken the medication for seven days or more. The index date was defined as the first day of drug treatment. The PPIs prescribed were rabeprazole, pantoprazole, omeprazole, lansoprazole, ilaprazole, esomeprazole, and dexlansoprazole, while the $\mathrm{H}_{2}$ RAs were ranitidine, nizatidine, lafutidine, famotidine, and cimetidine. We excluded all patients who had been prescribed any other primary or secondary medication(s) (i.e., PPIs and $\mathrm{H}_{2} \mathrm{RAs}$ ) within 180 days of the index date. Continuous drug exposures were grouped if the gap between prescriptions was less than 30 days. Non-users were defined as the patients who were not prescribed acid-suppressants and without a history of COVID-19 diagnosis within 180 days before the index date.

\section{Outcomes}

The primary outcome was defined as diagnosis of COVID-19 and the secondary outcomes were complications of COVID-19, namely, (1) all-cause mortality, (2) hospitalization with at least one of the following diagnoses, i.e., pneumonia, acute respiratory disease syndrome (ARDS), sepsis, or acute kidney 
injury (AKI), and (3) requirement of intensive respiratory interventions such as mechanical ventilation, extracorporeal membrane oxygenation procedure (ECMO), or tracheostomy.

\section{Statistical analyses}

We used propensity score matching (PSM) with regularized logistic regression models to balance baseline characteristics of the groups within the study cohort. ${ }^{17,18}$ Three different methods of analysis were used, viz., (1) unadjusted analysis, (2) one-to-four PSM with greedy nearest method, and (3) propensity score stratification with five strata. ${ }^{19}$

The covariates used include age, sex, all medication(s), medical procedure(s) and disease history, and comorbidity index in the database. To estimate the association between exposure and outcome(s), Cox proportional hazards regression models were used to compare incidence risk of COVID-19 between the cohorts. Patients were censored if they were no longer observable in the database. Data analyses were performed for three different cohort comparisons- (1) PPI users versus $\mathrm{H}_{2} R A$ users, (2) PPI users versus non-users who had been prescribed at least one medication other than acid-suppressants, and (3) $\mathrm{H}_{2} \mathrm{RA}$ users versus non-users who had been prescribed at least one medication other than acid-suppressants.

During secondary analysis, only patients with a definite diagnosis of COVID-19 were included and we measured complications due to COVID-19 disease among infected patients who had been prescribed acid-suppressive agents (i.e., PPIs and $\mathrm{H}_{2}$ RAs); other cohort comparisons were identical to that used during primary analysis, i.e., PPI users, $\mathrm{H}_{2}$ RA users, and non-users. Overall, six different cohort settings were applied during secondary analysis.

As there could be the residual bias in observational studies due to unmeasured covariates, we employed 123 falsification end points to estimate systematic error in the models, ${ }^{20,}{ }^{21}$ and that these outcomes were found to not affected by acid-suppressant use (Supplementary Table 1). The final hazard ratio (HR) and $95 \% \mathrm{Cls}$ were reported through empirical calibrations to adjust measured systematic errors from the analysis of 123 falsification end points. ${ }^{22}$

\section{Results}

\section{PPI use and risk of COVID-19}

\section{Baseline characteristics of the study population}

Of the 234,427 patients in the HIRA COVID-19 database, we finally included 26,166 patients prescribed $\mathrm{PPI}$ and 62,117 patients prescribed $\mathrm{H}_{2} \mathrm{RA}$ (Figure 1) with a person-years follow-up duration of 2,361 days for PPI users and 3,674 days for $\mathrm{H}_{2}$ RA users. Median follow-up days for PPI users was 14 (interquartile range [IQR], 7-75) while it was 7 for $\mathrm{H}_{2} \mathrm{RA}$ users (IQR, 7-34). Baseline characteristics of the primary analysis are listed in Table 1, which also provides standardized mean differences before and after PSM 
for the study population. Overall, 20,202 covariates were used for matching (Supplementary Figure 1), and among overall matched covariates, absolute standardized differences after PSM were less than 0.1 for $96.04 \%$ of the covariates, implying that the cohorts were adequately matched and therefore, comparable. Covariates with standardized differences greater than 0.1 after PSM were predominantly medications associated with acid-related disorders and anti-inflammatory products (e.g., bismuth oxide and sucralfate). 
Table 1

Baseline characteristics of patients with PPI and $\mathrm{H}_{2} \mathrm{RA}$ in the primary analysis.

\begin{tabular}{|c|c|c|c|c|c|c|}
\hline \multirow[t]{2}{*}{ Characteristics, \% } & \multicolumn{3}{|c|}{ Before propensity score matching } & \multicolumn{3}{|c|}{ After propensity score matching } \\
\hline & $\begin{array}{l}\begin{array}{l}\text { PPI } \\
\text { users }\end{array} \\
(n= \\
26,166)\end{array}$ & $\begin{array}{l}\mathrm{H}_{2} \mathrm{RA} \\
\text { users } \\
(\mathrm{n}= \\
62,629)\end{array}$ & $\begin{array}{l}\text { Absolute } \\
\text { standardized } \\
\text { difference }\end{array}$ & $\begin{array}{l}\begin{array}{l}\text { PPI } \\
\text { users }\end{array} \\
(n= \\
26,166)\end{array}$ & $\begin{array}{l}\mathrm{H}_{2} \mathrm{RA} \\
\text { users } \\
(\mathrm{n}= \\
62,117)\end{array}$ & $\begin{array}{l}\text { Absolute } \\
\text { standardized } \\
\text { difference }\end{array}$ \\
\hline \multicolumn{7}{|l|}{ Age group, $\%$} \\
\hline $18-19$ & 0.9 & 1.8 & 0.08 & 1.3 & 1.6 & 0.02 \\
\hline $20-24$ & 4.7 & 8.3 & 0.15 & 6.5 & 7.4 & 0.03 \\
\hline $25-29$ & 6.7 & 9.3 & 0.10 & 8.3 & 8.6 & 0.01 \\
\hline $30-34$ & 6.3 & 8.0 & 0.07 & 7.7 & 7.4 & 0.01 \\
\hline $35-39$ & 8.0 & 8.5 & 0.02 & 8.6 & 8.3 & 0.01 \\
\hline $40-44$ & 7.9 & 6.7 & 0.05 & 7.3 & 7.0 & 0.01 \\
\hline $45-49$ & 8.9 & 7.6 & 0.05 & 8.2 & 8.0 & 0.01 \\
\hline $50-54$ & 8.8 & 7.2 & 0.06 & 7.9 & 7.6 & 0.01 \\
\hline $55-59$ & 8.9 & 7.9 & 0.04 & 8.3 & 8.3 & $<0.01$ \\
\hline $60-64$ & 8.3 & 7.1 & 0.04 & 7.5 & 7.5 & $<0.01$ \\
\hline $65-69$ & 7.0 & 5.8 & 0.05 & 6.2 & 6.1 & $<0.01$ \\
\hline $70-74$ & 6.5 & 5.4 & 0.05 & 5.7 & 5.7 & $<0.01$ \\
\hline $75-79$ & 6.9 & 6.3 & 0.02 & 6.5 & 6.4 & $<0.01$ \\
\hline $80-84$ & 5.8 & 5.7 & $<0.01$ & 5.6 & 5.7 & $<0.01$ \\
\hline $85-89$ & 3.2 & 3.1 & 0.01 & 3.1 & 3.1 & $<0.01$ \\
\hline Gender, female, \% & 49.6 & 53.8 & 0.08 & 52.0 & 52.7 & 0.02 \\
\hline \multicolumn{7}{|l|}{$\begin{array}{l}\text { Medical history: } \\
\text { general, \% }\end{array}$} \\
\hline $\begin{array}{l}\text { Acute respiratory } \\
\text { disease }\end{array}$ & 49.2 & 56.9 & 0.16 & 49.5 & 56.7 & 0.15 \\
\hline Chronic liver disease & 5.6 & 3.9 & 0.08 & 5.0 & 4.0 & 0.05 \\
\hline
\end{tabular}

Values are presented as proportion of the patients (\%). Abbreviation: proton pump inhibitor (PPI), histamione-2 receptor antagonist $\left(\mathrm{H}_{2} \mathrm{RA}\right)$. 


\begin{tabular}{|c|c|c|c|c|c|c|}
\hline \multirow[t]{2}{*}{ Characteristics, \% } & \multicolumn{3}{|c|}{ Before propensity score matching } & \multicolumn{3}{|c|}{ After propensity score matching } \\
\hline & $\begin{array}{l}\text { PPI } \\
\text { users } \\
(n= \\
26,166)\end{array}$ & $\begin{array}{l}\mathrm{H}_{2} \mathrm{RA} \\
\text { users } \\
(\mathrm{n}= \\
62,629)\end{array}$ & $\begin{array}{l}\text { Absolute } \\
\text { standardized } \\
\text { difference }\end{array}$ & $\begin{array}{l}\text { PPI } \\
\text { users } \\
(n= \\
26,166)\end{array}$ & $\begin{array}{l}\mathrm{H}_{2} \mathrm{RA} \\
\text { users } \\
(\mathrm{n}= \\
62,117)\end{array}$ & $\begin{array}{l}\text { Absolute } \\
\text { standardized } \\
\text { difference }\end{array}$ \\
\hline $\begin{array}{l}\text { Chronic obstructive } \\
\text { lung disease }\end{array}$ & 4.6 & 3.7 & 0.05 & 4.1 & 3.9 & 0.01 \\
\hline Depressive disorder & 12.3 & 11.9 & 0.01 & 11.5 & 12.1 & 0.02 \\
\hline Diabetes mellitus & 17.4 & 14.6 & 0.08 & 15.8 & 15.1 & 0.02 \\
\hline $\begin{array}{l}\text { Gastrointestinal } \\
\text { hemorrhage }\end{array}$ & 3.5 & 1.9 & 0.10 & 3.3 & 1.9 & 0.09 \\
\hline Hyperlipidemia & 38.6 & 30.9 & 0.16 & 35.7 & 31.7 & 0.08 \\
\hline Hypertensive disorder & 35.4 & 29.7 & 0.12 & 35.7 & 31.7 & 0.08 \\
\hline Lesion of liver & 4.7 & 3.0 & 0.09 & 4.2 & 3.2 & 0.06 \\
\hline Osteoarthritis & 11.5 & 15.9 & 0.13 & 10.9 & 16.1 & 0.15 \\
\hline Pneumonia & 7.4 & 6.3 & 0.04 & 6.6 & 6.6 & $<0.00$ \\
\hline Renal impairment & 7.2 & 3.7 & 0.16 & 6.2 & 3.8 & 0.11 \\
\hline Rheumatoid arthritis & 2.2 & 2.2 & 0.00 & 2.0 & 2.2 & 0.02 \\
\hline Ulcerative colitis & 0.2 & 0.2 & $<0.01$ & 0.2 & 0.2 & $<0.01$ \\
\hline Atrial fibrillation & 2.6 & 1.5 & 0.07 & 2.3 & 1.6 & 0.05 \\
\hline $\begin{array}{l}\text { Cerebrovascular } \\
\text { disease }\end{array}$ & 5.2 & 4.8 & 0.02 & 4.7 & 4.9 & 0.01 \\
\hline Heart disease & 18.6 & 12.7 & 0.16 & 16.8 & 13.1 & 0.10 \\
\hline Heart failure & 7.9 & 4.8 & 0.12 & 7.0 & 5.1 & 0.08 \\
\hline Ischemic heart disease & 10.1 & 6.5 & 0.13 & 9.0 & 6.7 & 0.08 \\
\hline $\begin{array}{l}\text { Peripheral vascular } \\
\text { disease }\end{array}$ & 9.9 & 9.6 & 0.01 & 9.0 & 9.8 & 0.03 \\
\hline $\begin{array}{l}\text { Malignant neoplastic } \\
\text { disease }\end{array}$ & 9.0 & 7.6 & 0.05 & 8.1 & 8.0 & $<0.01$ \\
\hline
\end{tabular}

Values are presented as proportion of the patients (\%). Abbreviation: proton pump inhibitor (PPI), histamione-2 receptor antagonist $\left(\mathrm{H}_{2} \mathrm{RA}\right)$. 


\begin{tabular}{|c|c|c|c|c|c|c|}
\hline \multirow[t]{3}{*}{ Characteristics, \% } & \multicolumn{3}{|c|}{ Before propensity score matching } & \multicolumn{3}{|c|}{ After propensity score matching } \\
\hline & \multirow{2}{*}{$\begin{array}{l}\text { PPI } \\
\text { users } \\
(n= \\
26,166)\end{array}$} & \multirow{2}{*}{$\begin{array}{l}\mathrm{H}_{2} \mathrm{RA} \\
\text { users } \\
(\mathrm{n}= \\
62,629)\end{array}$} & \multirow[t]{2}{*}{$\begin{array}{l}\text { Absolute } \\
\text { standardized } \\
\text { difference }\end{array}$} & \multirow{2}{*}{$\begin{array}{l}\text { PPI } \\
\text { users } \\
(n= \\
26,166)\end{array}$} & \multirow{2}{*}{$\begin{array}{l}\mathrm{H}_{2} \mathrm{RA} \\
\text { users } \\
(\mathrm{n}= \\
62,117)\end{array}$} & \multirow[t]{2}{*}{$\begin{array}{l}\text { Absolute } \\
\text { standardized } \\
\text { difference }\end{array}$} \\
\hline & & & & & & \\
\hline $\begin{array}{l}\text { Antibacterials for } \\
\text { systemic use }\end{array}$ & 58.2 & 69.5 & 0.24 & 57.4 & 69.4 & 0.25 \\
\hline Antidepressants & 12.9 & 12.5 & 0.01 & 12.0 & 12.8 & 0.02 \\
\hline Antiepileptics & 11.1 & 12.2 & 0.04 & 9.9 & 12.6 & 0.09 \\
\hline $\begin{array}{l}\text { Anti-inflammatory and } \\
\text { antirheumatic } \\
\text { products }\end{array}$ & 49.2 & 68.4 & 0.40 & 49.2 & 68.2 & 0.39 \\
\hline Antineoplastic agents & 3.2 & 2.6 & 0.40 & 2.8 & 2.8 & $<0.00$ \\
\hline Antithrombotic agents & 38.4 & 46.1 & 0.16 & 36.9 & 46.3 & 0.19 \\
\hline Diuretics & 16.0 & 13.0 & 0.08 & 14.4 & 13.5 & 0.03 \\
\hline $\begin{array}{l}\text { Drugs for obstructive } \\
\text { airway disease }\end{array}$ & 17.8 & 21.1 & 0.08 & 17.2 & 21.4 & 0.11 \\
\hline Drugs used in diabetes & 16.2 & 12.9 & 0.09 & 14.6 & 13.4 & 0.03 \\
\hline Immunosuppressants & 3.5 & 2.6 & 0.05 & 3.1 & 2.6 & 0.03 \\
\hline Lipid modifying agents & 27.9 & 21.3 & 0.16 & 25.3 & 22.0 & 0.08 \\
\hline Opioids & 50.4 & 61.3 & 0.22 & 29.2 & 61.6 & 0.25 \\
\hline $\begin{array}{l}\text { Charlson comorbidity } \\
\text { index }\end{array}$ & 2.79 & 2.79 & 0.17 & 2.32 & 2.61 & 0.06 \\
\hline $\begin{array}{l}\text { Values are presented a } \\
\text { histamione- } 2 \text { receptor }\end{array}$ & portic & he pat & (\%). Abbr & : pro & ump int & or (PPI), \\
\hline
\end{tabular}

\section{Association of PPI use and risk of COVID-19}

Table 2 show the results of the primary analysis that estimated the association between PPI or $\mathrm{H}_{2} \mathrm{RA}$ usage and risk of COVID-19. PSM-unadjusted analysis showed that PPI use was not significantly associated with risk of COVID-19 infection compared to $\mathrm{H}_{2}$ RA use (calibrated HR, 1.49 [95\% Cl, 0.663.36]), moreover, PSM adjusted analyses revealed that PPI use was not significantly associated with lower risk of COVID-19 infection in one-to-four PSM analysis (calibrated HR, 0.81 [95\% Cl, 0.30-2.19]) and in stratification of propensity scores analysis (calibrated HR, 1.03 [95\% $\mathrm{Cl}, 0.51-2.08]$ ). 
Table 2

The associations of the risk of COVID-19 infection between PPI and $\mathrm{H}_{2} \mathrm{RA}$ users

\begin{tabular}{|c|c|c|c|c|c|}
\hline \multirow[t]{2}{*}{ Analysis settings } & \multicolumn{2}{|c|}{ No. of subjects } & \multicolumn{2}{|c|}{ No. of outcome occurrence } & \multirow{2}{*}{$\begin{array}{l}\text { calibrated HR } \\
{[95 \% \mathrm{Cl}]}\end{array}$} \\
\hline & PPI users & $\mathrm{H}_{2} \mathrm{RA}$ users & PPI users & $\mathrm{H}_{2} \mathrm{RA}$ users & \\
\hline \multirow[t]{2}{*}{ Unadjusted } & 26,166 & 62,629 & 96 & 104 & 1.49 \\
\hline & & & & & $(0.66-3.36)$ \\
\hline \multirow[t]{2}{*}{ Stratification } & 26,166 & 62,629 & 96 & 104 & 1.03 \\
\hline & & & & & $(0.51-2.08)$ \\
\hline \multirow[t]{2}{*}{ 1:4 matching } & 26,166 & 62,117 & 96 & 104 & 0.81 \\
\hline & & & & & $(0.30-2.19)$ \\
\hline \multirow[t]{2}{*}{ Analysis settings } & \multicolumn{2}{|c|}{ No. of subjects } & \multicolumn{2}{|c|}{ No. of outcome occurrence } & calibrated HR \\
\hline & PPI users & Non-users & PPI users & Non-users & {$[95 \% \mathrm{Cl}]$} \\
\hline \multirow[t]{2}{*}{ Unadjusted } & 26,044 & 74,975 & 113 & 3,012 & 0.43 \\
\hline & & & & & $(0.11-1.61)$ \\
\hline \multirow[t]{2}{*}{ Stratification } & 26,044 & 74,975 & 113 & 3,012 & 0.50 \\
\hline & & & & & $(0.17-1.52)$ \\
\hline \multirow[t]{2}{*}{ 1:4 matching } & 22,765 & 71,408 & 111 & 2,848 & 0.47 \\
\hline & & & & & $(0.17-1.29)$ \\
\hline \multirow[t]{2}{*}{ Analysis settings } & \multicolumn{2}{|c|}{ No. of subjects } & \multicolumn{2}{|c|}{ No. of outcome occurrences } & calibrated HR \\
\hline & $\mathrm{H}_{2} \mathrm{RA}$ users & Non-users & $\mathrm{H}_{2} \mathrm{RA}$ users & Non-users & {$[95 \% \mathrm{Cl}]$} \\
\hline \multirow[t]{2}{*}{ Unadjusted } & 51,545 & 64,013 & 112 & 2,616 & 0.30 \\
\hline & & & & & $(0.09-0.96)$ \\
\hline \multirow[t]{2}{*}{ Stratification } & 51,545 & 64,013 & 112 & 2,616 & 0.46 \\
\hline & & & & & $(0.15-1.43)$ \\
\hline \multirow[t]{2}{*}{$1: 4$ matching } & 29,845 & 64,013 & 107 & 2,616 & 0.48 \\
\hline & & & & & $(0.17-1.37)$ \\
\hline
\end{tabular}

A comparison of COVID-19 between acid-suppressant users and non-users revealed that PPI use was not significantly associated with lower risk of COVID-19 compared to non-users before adjusted analysis 
(calibrated HR, 0.43 [95\% Cl, 0.11-1.61]), after one-to-four matched analysis (calibrated $\mathrm{HR}, 0.47$ [95\% Cl, 0.17-1.29]), and stratification analysis (calibrated $\mathrm{HR}, 0.50$ [95\% $\mathrm{Cl}, 0.17-1.52]$ ). Among $\mathrm{H}_{2} \mathrm{RA}$ users, medication was associated with the lower risk of COVID-19 compared to non-users during unadjusted analysis (calibrated $\mathrm{HR}, 0.30$ [95\% $\mathrm{Cl}, 0.09-0.96]$ ). In other analyses, $\mathrm{H}_{2} \mathrm{RA}$ use was not significantly associated with infection despite one-to-four PSM (calibrated HR, 0.48 [95\% $\mathrm{Cl}, 0.17-1.37]$ ) or propensity score stratification (calibrated $\mathrm{HR}, 0.46$ [95\% Cl, 0.15-1.43]).

\section{PPI use and complications of COVID-19 disease Baseline characteristics of the study population}

Secondary analysis was performed with data from 1,260 patients diagnosed with COVID-19; of these, 410 patients were prescribed PPI and 804 were given $\mathrm{H}_{2}$ RA medication. Subjects were matched based on sex, age groups, medical history (chronic obstructive lung disease and chronic kidney disease), and the Charlson comorbidity index, and the absolute standardized mean difference for all covariates after PSM was less than 0.2. Baseline characteristics are presented in Table 3. 
Table 3

Baseline characteristics of COVID-19 patients with PPI and $\mathrm{H}_{2} \mathrm{RA}$ in the secondary analysis.

\begin{tabular}{|c|c|c|c|c|c|c|}
\hline \multirow[t]{3}{*}{ Characteristics, \% } & \multicolumn{3}{|c|}{ Before propensity score matching } & \multicolumn{3}{|c|}{ After propensity score matching } \\
\hline & $\begin{array}{l}\text { PPI } \\
\text { users }\end{array}$ & $\begin{array}{l}\mathrm{H}_{2} \mathrm{RA} \\
\text { users }\end{array}$ & $\begin{array}{l}\text { Absolute } \\
\text { standardized } \\
\text { difference }\end{array}$ & $\begin{array}{l}\text { PPI } \\
\text { users }\end{array}$ & $\begin{array}{l}\mathrm{H}_{2} \mathrm{RA} \\
\text { users }\end{array}$ & $\begin{array}{l}\text { Absolute } \\
\text { standardized } \\
\text { difference }\end{array}$ \\
\hline & $\begin{array}{l}(n= \\
420)\end{array}$ & $\begin{array}{l}(n= \\
840)\end{array}$ & & $\begin{array}{l}(n= \\
410)\end{array}$ & $\begin{array}{l}(n= \\
804)\end{array}$ & \\
\hline
\end{tabular}

Age group, \%

\begin{tabular}{|lllllll}
\hline $18-19$ & $<1.2$ & 1.7 & 0.06 & $<1.2$ & 1.7 & 0.06 \\
\hline $20-24$ & 4.8 & 10.4 & 0.21 & 4.9 & 4.8 & 0.01 \\
\hline $25-29$ & 4.5 & 8.1 & 0.15 & 4.6 & 4.3 & 0.02 \\
\hline $30-34$ & 4.0 & 2.9 & 0.06 & 4.1 & 4.0 & 0.01 \\
\hline $35-39$ & 2.6 & 7.0 & 0.21 & 2.7 & 2.8 & 0.01 \\
\hline $40-44$ & 3.1 & 5.2 & 0.11 & 3.2 & 3.7 & 0.03 \\
\hline $45-49$ & 5.7 & 7.5 & 0.07 & 5.9 & 4.7 & 0.05 \\
\hline $50-54$ & 12.1 & 8.3 & 0.13 & 12.2 & 11.2 & 0.03 \\
\hline $55-59$ & 12.1 & 10.7 & 0.04 & 12.4 & 12.2 & 0.01 \\
\hline $60-64$ & 16.7 & 10.1 & 0.19 & 16.3 & 13.1 & 0.09 \\
\hline $65-69$ & 10.2 & 7.3 & 0.10 & 10.0 & 9.6 & 0.01 \\
\hline $70-74$ & 6.9 & 6.4 & 0.02 & 6.8 & 7.8 & 0.04 \\
\hline $75-79$ & 7.1 & 6.5 & 0.02 & 7.3 & 8.9 & 0.06 \\
\hline $80-84$ & 4.5 & 3.8 & 0.04 & 4.4 & 5.2 & 0.04 \\
\hline $85-89$ & 3.3 & 3.0 & 0.02 & 2.9 & 4.4 & 0.08 \\
\hline Gender: female, \% & 60.7 & 66.8 & 0.08 & 61.0 & 65.9 & 0.02 \\
\hline $\begin{array}{l}\text { Medical history: } \\
\text { general, \% }\end{array}$ & & & & & & \\
\hline
\end{tabular}

$\begin{array}{lllllll}\text { Chronic obstructive } & <0.1 & <0.1 & <0.1 & <0.1 & 0.12 & 0.09\end{array}$ lung disease

$\begin{array}{lllllll}\text { Chronic kidney } & <0.1 & <0.1 & 0.11 & <0.1 & <0.1 & 0.09\end{array}$
disease 


\begin{tabular}{|c|c|c|c|c|c|c|}
\hline \multirow[t]{3}{*}{ Characteristics, \% } & \multicolumn{3}{|c|}{ Before propensity score matching } & \multicolumn{3}{|c|}{ After propensity score matching } \\
\hline & $\begin{array}{l}\text { PPI } \\
\text { users }\end{array}$ & $\begin{array}{l}\mathrm{H}_{2} \mathrm{RA} \\
\text { users }\end{array}$ & $\begin{array}{l}\text { Absolute } \\
\text { standardized } \\
\text { difference }\end{array}$ & $\begin{array}{l}\text { PPI } \\
\text { users }\end{array}$ & $\begin{array}{l}\mathrm{H}_{2} \mathrm{RA} \\
\text { users }\end{array}$ & $\begin{array}{l}\text { Absolute } \\
\text { standardized } \\
\text { difference }\end{array}$ \\
\hline & $\begin{array}{l}(n= \\
420)\end{array}$ & $\begin{array}{l}(n= \\
840)\end{array}$ & & $\begin{array}{l}(n= \\
410)\end{array}$ & $\begin{array}{l}(n= \\
804)\end{array}$ & \\
\hline $\begin{array}{l}\text { Charlson } \\
\text { comorbidity index }\end{array}$ & 3.0 & 2.3 & 0.27 & 3.0 & 2.7 & 0.1 \\
\hline
\end{tabular}

Values are presented as proportion of the patients (\%). Abbreviation: SARS-CoV-2 infection (COVID19), proton pump inhibitor (PPI), histamione-2 receptor antagonist $\left(\mathrm{H}_{2} \mathrm{RA}\right)$

\section{Association between PPI use and complications of COVID- 19 disease}

Table 4 provides the results of the secondary analysis, which showed no significant association between PPI or $\mathrm{H}_{2}$ RA use and all-cause mortality among COVID-19 patients, i.e., (1) unadjusted analysis (calibrated HR, 0.82 [95\% $\mathrm{Cl}, 0.47-1.41]$ ), or (2) propensity score stratification (calibrated HR, 0.63 [95\% $\mathrm{Cl}, 0.37-1.07])$, however, (3) one-to-four PSM analysis showed significant associated between PPI use and all-cause mortality (calibrated $\mathrm{HR}, 0.54$ [95\% Cl, 0.31-0.95]). Further, hospitalization with pneumonia, ARDS, sepsis, or AKI were not associated with PPI or $\mathrm{H}_{2} \mathrm{RA}$ use, irrespective of the type of analysis, i.e., (1) unadjusted analysis (calibrated $\mathrm{HR}, 1.12$ [95\% $\mathrm{Cl}, 0.87-1.43]$ ), (2) propensity score stratification (calibrated HR, 0.96 [95\% Cl, 0.79-1.17]), or (3) one-to-four PSM (calibrated HR, 0.88 [95\% Cl, 0.72-1.08]). Similarly, there was no association between PPI and requirement for intensive respiratory interventions, (1) unadjusted analysis (calibrated $\mathrm{HR}, 1.28$ [95\% Cl, 0.65-2.50]), (2) propensity score stratification (calibrated HR, 1.01 [95\% Cl, 0.52-1.97]), and (3) one-to-four PSM (calibrated HR, 0.92 [95\% Cl, 0.461.82]). The results for hospitalized COVID-19 patients are provided in Table 5. 
Table 4

The associations of the clinical outcomes between PPI and $\mathrm{H}_{2} \mathrm{RA}$ users among COVID-19 diagnosed patients.

\begin{tabular}{|c|c|c|c|c|c|c|c|}
\hline \multirow[t]{2}{*}{ Outcome } & \multirow[t]{2}{*}{$\begin{array}{l}\text { Analysis } \\
\text { settings }\end{array}$} & \multicolumn{2}{|c|}{$\begin{array}{l}\text { No. of } \\
\text { subjects }\end{array}$} & \multicolumn{3}{|c|}{$\begin{array}{l}\text { No. of outcome } \\
\text { occurrence }\end{array}$} & \multirow{2}{*}{$\begin{array}{l}\text { calibrated } \\
\text { HR } \\
{[95 \% \mathrm{Cl}]}\end{array}$} \\
\hline & & $\begin{array}{l}\text { PPI } \\
\text { users }\end{array}$ & $\begin{array}{l}\mathrm{H}_{2} \mathrm{RA} \\
\text { users }\end{array}$ & PPI users & & & \\
\hline \multirow[t]{6}{*}{ Covid-19 death } & \multirow[t]{2}{*}{ Unadjusted } & \multirow[t]{2}{*}{420} & \multirow[t]{2}{*}{840} & \multirow[t]{2}{*}{21} & \multirow{2}{*}{\multicolumn{2}{|c|}{40}} & 0.82 \\
\hline & & & & & & & $\begin{array}{l}(0.47- \\
1.41)\end{array}$ \\
\hline & \multirow[t]{2}{*}{ Stratification } & \multirow[t]{2}{*}{420} & \multirow[t]{2}{*}{840} & \multirow[t]{2}{*}{21} & \multirow{2}{*}{\multicolumn{2}{|c|}{40}} & 0.63 \\
\hline & & & & & & & $\begin{array}{l}(0.37- \\
1.07)\end{array}$ \\
\hline & \multirow{2}{*}{$\begin{array}{l}1: 4 \\
\text { matching }\end{array}$} & \multirow[t]{2}{*}{410} & \multirow[t]{2}{*}{804} & \multirow[t]{2}{*}{19} & \multirow{2}{*}{\multicolumn{2}{|c|}{40}} & 0.54 \\
\hline & & & & & & & $\begin{array}{l}(0.31- \\
0.95)\end{array}$ \\
\hline \multirow[t]{2}{*}{ Outcome } & \multirow[t]{2}{*}{$\begin{array}{l}\text { Analysis } \\
\text { settings }\end{array}$} & \multicolumn{2}{|c|}{$\begin{array}{l}\text { No. of } \\
\text { subjects }\end{array}$} & \multicolumn{3}{|c|}{$\begin{array}{l}\text { No. of outcome } \\
\text { occurrence }\end{array}$} & $\begin{array}{l}\text { calibrated } \\
\text { HR }\end{array}$ \\
\hline & & $\begin{array}{l}\text { PPI } \\
\text { users }\end{array}$ & $\begin{array}{l}\mathrm{H}_{2} \mathrm{RA} \\
\text { users }\end{array}$ & PPI us & & $\begin{array}{l}\mathrm{H}_{2} \mathrm{RA} \\
\text { users }\end{array}$ & [95\% Cl] \\
\hline \multirow{6}{*}{$\begin{array}{l}\text { Hospitalization with } \\
\text { pneumonia, ARDS, sepsis, } \\
\text { and AKI }\end{array}$} & \multirow[t]{2}{*}{ Unadjusted } & \multirow[t]{2}{*}{393} & \multirow[t]{2}{*}{789} & \multirow{2}{*}{\multicolumn{3}{|c|}{164}} & 1.12 \\
\hline & & & & & & & $\begin{array}{l}(0.87- \\
1.43)\end{array}$ \\
\hline & \multirow[t]{2}{*}{ Stratification } & \multirow[t]{2}{*}{393} & \multirow[t]{2}{*}{789} & \multirow[t]{2}{*}{164} & & \multirow[t]{2}{*}{262} & 0.96 \\
\hline & & & & & & & $\begin{array}{l}(0.79- \\
1.17)\end{array}$ \\
\hline & \multirow{2}{*}{$\begin{array}{l}1: 4 \\
\text { matching }\end{array}$} & 382 & 754 & 160 & & 259 & 0.88 \\
\hline & & & & & & & $\begin{array}{l}(0.72- \\
1.08)\end{array}$ \\
\hline Outcome & $\begin{array}{l}\text { Analysis } \\
\text { settings }\end{array}$ & No. of $s$ & ubjects & $\begin{array}{l}\text { No. o } \\
\text { occur }\end{array}$ & & me & $\begin{array}{l}\text { calibrated } \\
\text { HR }\end{array}$ \\
\hline & & $\begin{array}{l}\text { PPI } \\
\text { users }\end{array}$ & $\begin{array}{l}\mathrm{H}_{2} \mathrm{RA} \\
\text { users }\end{array}$ & PPI u & & $\begin{array}{l}\mathrm{H}_{2} \mathrm{RA} \\
\text { users }\end{array}$ & [95\% Cl] \\
\hline
\end{tabular}

Abbreviation: SARS-Cov-2 (COVID-19), proton pump inhibitor (PPI), histamione-2 receptor antagonist $\left(\mathrm{H}_{2} \mathrm{RA}\right)$, hazard ratio $(\mathrm{HR})$, confidence interval $(\mathrm{Cl})$, acute respiratory distress syndrome (ARDS), acute kidney injury (AKI) 


\begin{tabular}{|c|c|c|c|c|c|c|}
\hline \multirow[t]{5}{*}{$\begin{array}{l}\text { Occurrence of a composite } \\
\text { intensive respiratory } \\
\text { intervention }\end{array}$} & Unadjusted & 411 & 831 & 16 & 20 & $\begin{array}{l}1.28 \\
(0.65- \\
2.50)\end{array}$ \\
\hline & \multirow[t]{2}{*}{ Stratification } & \multirow[t]{2}{*}{411} & \multirow[t]{2}{*}{831} & \multirow[t]{2}{*}{16} & \multirow[t]{2}{*}{20} & 1.01 \\
\hline & & & & & & $\begin{array}{l}(0.52- \\
1.97)\end{array}$ \\
\hline & \multirow{2}{*}{$\begin{array}{l}\text { 1:4 } \\
\text { matching }\end{array}$} & \multirow[t]{2}{*}{402} & \multirow[t]{2}{*}{795} & \multirow[t]{2}{*}{15} & \multirow[t]{2}{*}{20} & 0.92 \\
\hline & & & & & & $\begin{array}{l}(0.46- \\
1.82)\end{array}$ \\
\hline
\end{tabular}

Abbreviation: SARS-Cov-2 (COVID-19), proton pump inhibitor (PPI), histamione-2 receptor antagonist $\left(\mathrm{H}_{2} \mathrm{RA}\right)$, hazard ratio $(\mathrm{HR})$, confidence interval $(\mathrm{Cl})$, acute respiratory distress syndrome (ARDS), acute kidney injury (AKI) 
Table 5

Secondary analysis results among hospitalized patients due to COVID-19

\begin{tabular}{|c|c|c|c|c|c|c|}
\hline \multirow[t]{2}{*}{ Outcome } & \multirow[t]{2}{*}{$\begin{array}{l}\text { Analysis } \\
\text { settings }\end{array}$} & \multicolumn{2}{|c|}{$\begin{array}{l}\text { No. of } \\
\text { subjects }\end{array}$} & \multicolumn{2}{|c|}{$\begin{array}{l}\text { No. of } \\
\text { outcome } \\
\text { occurrence }\end{array}$} & \multirow{2}{*}{$\begin{array}{l}\text { calibrated } \\
\text { HR } \\
{[95 \% \mathrm{Cl}]}\end{array}$} \\
\hline & & $\begin{array}{l}\text { PPI } \\
\text { users }\end{array}$ & $\begin{array}{l}\mathrm{H}_{2} \mathrm{RA} \\
\text { users }\end{array}$ & $\begin{array}{l}\text { PPI } \\
\text { users }\end{array}$ & $\begin{array}{l}\mathrm{H}_{2} \mathrm{RA} \\
\text { users }\end{array}$ & \\
\hline \multirow[t]{6}{*}{ All-cause mortality } & \multirow[t]{2}{*}{ Unadjusted } & \multirow[t]{2}{*}{371} & \multirow[t]{2}{*}{747} & \multirow[t]{2}{*}{12} & \multirow[t]{2}{*}{30} & 0.60 \\
\hline & & & & & & $\begin{array}{l}(0.30- \\
1.20)\end{array}$ \\
\hline & \multirow[t]{2}{*}{ Stratification } & \multirow[t]{2}{*}{371} & \multirow[t]{2}{*}{747} & \multirow[t]{2}{*}{12} & \multirow[t]{2}{*}{30} & 0.50 \\
\hline & & & & & & $\begin{array}{l}(0.26- \\
0.99)\end{array}$ \\
\hline & \multirow{2}{*}{$\begin{array}{l}\text { 1:4 } \\
\text { matching* }\end{array}$} & \multirow[t]{2}{*}{366} & \multirow[t]{2}{*}{719} & \multirow[t]{2}{*}{11} & \multirow[t]{2}{*}{30} & \\
\hline & & & & & & $\begin{array}{l}(0.26- \\
1.06)\end{array}$ \\
\hline \multirow[t]{2}{*}{ Outcome } & \multirow[t]{2}{*}{$\begin{array}{l}\text { Analysis } \\
\text { settings }\end{array}$} & \multicolumn{2}{|c|}{$\begin{array}{l}\text { No. of } \\
\text { subjects }\end{array}$} & \multicolumn{2}{|c|}{$\begin{array}{l}\text { No. of } \\
\text { outcome } \\
\text { occurrence }\end{array}$} & $\begin{array}{l}\text { calibrated } \\
\text { HR }\end{array}$ \\
\hline & & $\begin{array}{l}\text { PPI } \\
\text { users }\end{array}$ & $\begin{array}{l}\mathrm{H}_{2} \mathrm{RA} \\
\text { users }\end{array}$ & $\begin{array}{l}\text { PPI } \\
\text { users }\end{array}$ & $\begin{array}{l}\mathrm{H}_{2} \mathrm{RA} \\
\text { users }\end{array}$ & \\
\hline \multirow{6}{*}{$\begin{array}{l}\text { Occurrence of a composite } \\
\text { intensive respiratory intervention }\end{array}$} & \multirow[t]{2}{*}{ Unadjusted } & \multirow[t]{2}{*}{364} & \multirow[t]{2}{*}{737} & \multirow[t]{2}{*}{10} & \multirow[t]{2}{*}{12} & 1.28 \\
\hline & & & & & & $\begin{array}{l}(0.54- \\
3.04)\end{array}$ \\
\hline & \multirow[t]{2}{*}{ Stratification } & \multirow[t]{2}{*}{364} & \multirow[t]{2}{*}{737} & \multirow[t]{2}{*}{10} & \multirow[t]{2}{*}{12} & 1.10 \\
\hline & & & & & & $\begin{array}{l}(0.47- \\
2.58)\end{array}$ \\
\hline & \multirow{2}{*}{$\begin{array}{l}1: 4 \\
\text { matching* }\end{array}$} & 358 & 709 & 9 & 12 & 0.98 \\
\hline & & & & & & $\begin{array}{l}(0.40- \\
2.39)\end{array}$ \\
\hline $\begin{array}{l}\text { Abbreviation: proton pump inhibit } \\
\text { confidence interval (CI) }\end{array}$ & PI), histamion & 2 recept & r antag & nist $\left(\mathrm{H}_{2}\right)$ & A), haza & ratio $(\mathrm{HR})$, \\
\hline
\end{tabular}

\section{Discussion}

This study aimed to estimate and compare risk of incidence and assess outcomes after COVID-19 in Korean patients prescribed PPI or $\mathrm{H}_{2} \mathrm{RA}$. Specifically, we evaluated the incidence of COVID 19 in subjects 
prescribed PPI or $\mathrm{H}_{2}$ RA for more than 7 days and show that short-term use of PPI was not associated with incidence of COVID-19 compared to short-term $\mathrm{H}_{2}$ RA users or non-users. Moreover, among COVID-19 patients, PPI use for $\geq 7$ days was not significantly associated with risk of complications compared to $\mathrm{H}_{2}$ RA use except for all-cause mortality in PSM analysis, and it was not associated with complications in hospitalized patients.

Two recent studies have addressed the association between PPI use and incidence of COVID-19 infection. ${ }^{11,12}$ Lee et al have reported that patients taking PPIs are at increased risk for severe clinical outcomes with COVID-19 but that they are not more susceptible to SARS- CoV-2 infection. ${ }^{12}$ They defined current PPI users as patients who took PPIs 1-30 days before the first SARS-CoV-2 test date. ${ }^{12}$ Further, they also showed that there was no significant difference in SARS-CoV-2 positivity rates between PPI users and non-users, irrespective of short-term ( $<30$ days) or long-term ( $>30$ days) use. ${ }^{12}$ Another study, an online survey by Almario et al., reported that individuals using PPIs up to once daily (aOR $2.15 ; 95 \% \mathrm{Cl}$, $1.90-2.44$ ) or twice daily (aOR $3.67 ; 95 \% \mathrm{Cl}, 2.93-4.60$ ) had significantly higher odds for testing COVID19 positive compared to those not taking PPIs. ${ }^{11}$ In contrast, we show that PPI use was not associated with the higher risk of COVID-19 infection compared to $\mathrm{H}_{2} \mathrm{RA}$ use or no acid-suppressant use. This could be due to our use of the Korean national claims database wherein data was converted to the OMOP-CDM format, and this permitted adjustment for many more covariates than previous studies. Additionally, large-scale propensity matching was used to overcome potentially unmeasured confounding factors and we also performed multiple sensitivity analyses. We also calibrated our analysis using 123 falsification end points to detect and reduce confounding factors, selection bias, and systematic errors. Thus, of the 48 analyses performed (9 primary and 39 secondary), most results were consistent with the calibrations.

In the secondary analysis, we compared the complication of COVID-19 between PPI and $\mathrm{H}_{2} \mathrm{RA}$ using multiple sensitivity analyses. The result showed no significant association between PPI and $\mathrm{H}_{2} \mathrm{RA}$. Only PSM analysis measuring the association between PPI and all-cause mortality, compared to $\mathrm{H}_{2} \mathrm{RA}$, showed significant results, however, the other analyses (i.e., unadjusted and stratification) showed opposite results. We could not perform large-scale PSM in the secondary analysis due to small number of included COVID-19 patients, therefore, there might be biases in the result.

To date, several studies have addressed clinical outcomes in COVID-19; however, most studies only included a small number of patients and were limited by the presence of confounding factors. ${ }^{12-14,23}$ Lee et al found that PPI use led to greater risk of severe clinical outcomes in COVID-19, including intensive care unit admission, requirement of invasive ventilation, or death. ${ }^{12}$ However, that study did not consider PPI use after COVID-19 diagnosis, and the comparator group comprised non-PPI users, which could have led to indication bias, i.e., patients in the PPI group could have experienced a more severe course of COVID-19 compared to non-users and the difference might have led to more severe outcomes. Therefore, to avoid indication bias, we compared clinical outcomes between PPI and $\mathrm{H}_{2} \mathrm{RA}$ users, and consistent with our results, Zhang et al also reported that PPI use had no effect on the clinical course of COVID-19. ${ }^{13}$ Additionally, Taştemur et al. have suggested that PPIs may be used for both prophylaxis and 
treatment because hydroxychloroquine and azithromycin may prevent viral spread by accumulating in organelles with acidic content and raising their $\mathrm{pH}$. Thus, given their effects on $\mathrm{pH}$, they concluded that PPIs show similar effects on viral entry and intracellular distribution. ${ }^{15}$ Such inconsistent results imply that the risk and benefits of PPI use in viral infection have remained controversial to date. ${ }^{23}$

Our study has certain limitations. First, although we used large-scale PSM in the primary analysis, there were a few relatively unmatched covariates that may have led to selection bias, showing standardized mean difference greater than 0.1. Additionally, there might have been residual indication bias between $\mathrm{PPI}$ and $\mathrm{H}_{2} \mathrm{RA}$ users. Nonetheless, we measured and adjusted the systematic error in this study through empirical calibration by employing 123 falsification end points in the primary analysis. Second, we only included PPI use for 7 days, and therefore, we could not evaluate the effects of long-term PPI use, and as the HIRA database also had data only pertaining to a short period, we could not analyze the long-term effects of acid-suppressants. Third, this was an observational study; therefore, it is not possible to establish causality. The effects of acid-suppressants on viral infection, especially COVID-19, require further clarification.

\section{Conclusions}

In this study, using large-scale PSM and multiple sensitivity analyses, we show that, compared to $\mathrm{H}_{2} \mathrm{RA}$ use, short-term PPI use is not associated with incidence of COVID-19 infection and severe clinical outcomes. Nevertheless, the effects of long-term PPI use on the incidence and clinical outcomes in COVID-19 disease need to be clearly established.

\section{Abbreviations}

PPI

proton pump inhibitor

COVID-19

SARS-CoV-2 infection

$\mathrm{H}_{2} \mathrm{RA}$

histamine-2 receptor antagonist

OR

odds ratio

$\mathrm{Cl}$

confidence interval

HIRA

Health Insurance Review and Assessment

OMOP

Observation Medical Outcomes Partnership

CDM

Page $17 / 21$ 
Common Data Model

ARDS

acute respiratory disease syndrome

AKI

acute kidney injury

ECMO

extracorporeal membrane oxygenation

PSM

propensity score matching

HR

hazard ratio

IQR

interquartile range

\section{Declarations}

\section{Ethics and approval and consent to participate}

This study is a retrospective cohort study not involving any human tissue data and was approved by the institutional review board of the Kangdong Sacred Heart Hospital (no. 2020-04-001). All methods were carried out in accordance with guidelines of out ethics committee, and informed consent was waived by ethics committee.

\section{Consent for publication}

Not applicable

\section{Availability of data and materials}

The datasets generated and analyzed during the current study are not publicly available since the Health Insurance Review and Assessment service is not providing dataset to public anymore. The result of analyzed data are available through contact with corresponding author's e-mail doctorssi@kdh.or.kr.

\section{Competing interest}

The authors declare that they have no competing interests.

\section{Funding}

This research was funded by the Bio Industrial Strategic Technology Development Program (20003883, 20005021) funded By the Ministry of Trade, Industry \& Energy (MOTIE, Korea) and a grant from the Korea Health Technology R\&D Project through the Korea Health Industry Development Institute (KHIDI), funded by the Ministry of Health \&Welfare, Republic of Korea (grant number: HI19C0143, HR16C0001). 


\section{Author's contribution}

JP, SCY, JC, RWP, SIS, CHP, WGS contributed study concept and design. JP contributed statistical analysis of the study. JP, SCY, JC, SIS, and RWP contributed analysis and interpretation of data, drafting of the manuscript. RWP, SIS, WGS contributed obtaining funding. RWP and SIS contributed supervision of the study. JP and SCY contributed equally to this work. RWP and SIS contributed equally to this work.

\section{Acknowledgement}

The authors thank the healthcare professionals dedicated to treating COVID-19 patients in South Korea, the Ministry of Health and Welfare, and the Health Insurance Review \& Assessment Service of Korea for sharing the national health insurance claims data in a prompt manner.

\section{References}

1. Savarino V, Tosetti C, Benedetto E, et al. Appropriateness in prescribing PPIs: A position paper of the Italian Society of Gastroenterology (SIGE) - Study section "Digestive Diseases in Primary Care". Digestive and liver disease: official journal of the Italian Society of Gastroenterology and the Italian Association for the Study of the Liver 2018;50(9):894-902. doi: 10.1016/j.dld.2018.07.004

2. Malfertheiner P, Kandulski A, Venerito M. Proton-pump inhibitors: understanding the complications and risks. Nat Rev Gastroenterol Hepato/2017;14(12):697-710. doi: 10.1038/nrgastro.2017.117

3. Vaezi MF, Yang YX, Howden CW. Complications of Proton Pump Inhibitor Therapy. Gastroenterology 2017;153(1):35-48. doi: 10.1053/j.gastro.2017.04.047

4. Gulmez SE, Holm A, Frederiksen $\mathrm{H}$, et al. Use of proton pump inhibitors and the risk of communityacquired pneumonia: a population-based case-control study. Archives of internal medicine 2007;167(9):950-5. doi: 10.1001/archinte.167.9.950 [published Online First: 2007/05/16]

5. Laheij RJ, Sturkenboom MC, Hassing RJ, et al. Risk of community-acquired pneumonia and use of gastric acid-suppressive drugs. Jama 2004;292(16):1955-60. doi: 10.1001/jama.292.16.1955 [published Online First: 2004/10/28]

6. Barkun AN, Bardou M, Pham CQ, et al. Proton pump inhibitors vs. histamine 2 receptor antagonists for stress-related mucosal bleeding prophylaxis in critically ill patients: a meta-analysis. The American journal of gastroenterology 2012;107(4):507-20; quiz 21. doi: 10.1038/ajg.2011.474 [published Online First: 2012/02/01]

7. Eom CS, Jeon CY, Lim JW, et al. Use of acid-suppressive drugs and risk of pneumonia: a systematic review and meta-analysis. CMAJ: Canadian Medical Association journal = journal de l'Association medicale canadienne 2011;183(3):310-9. doi: 10.1503/cmaj.092129 [published Online First: 2010/12/22]

8. Ho SW, Teng YH, Yang SF, et al. Association of Proton Pump Inhibitors Usage with Risk of Pneumonia in Dementia Patients. J Am Geriatr Soc 2017;65(7):1441-47. doi: 10.1111/jgs.14813 
9. MacLaren R, Reynolds PM, Allen RR. Histamine-2 receptor antagonists vs proton pump inhibitors on gastrointestinal tract hemorrhage and infectious complications in the intensive care unit. JAMA internal medicine 2014;174(4):564-74. doi: 10.1001/jamainternmed.2013.14673 [published Online First: 2014/02/19]

10. Othman F, Crooks CJ, Card TR. Community acquired pneumonia incidence before and after proton pump inhibitor prescription: population based study. BMJ 2016;355:i5813. doi: 10.1136/bmj.i5813

11. Almario CV, Chey WD, Spiegel BMR. Increased Risk of COVID-19 Among Users of Proton Pump Inhibitors. The American journal of gastroenterology 2020;115(10):1707-15. doi:

10.14309/ajg.0000000000000798 [published Online First: 2020/08/28]

12. Lee SW, Ha EK, Yeniova A, et al. Severe clinical outcomes of COVID-19 associated with proton pump inhibitors: a nationwide cohort study with propensity score matching. Gut 2021;70(1):76-84. doi: 10.1136/gutjnl-2020-322248 [published Online First: 2020/08/01]

13. Zhang XY, Li T, Wu H, et al. Analysis of the Effect of Proton-Pump Inhibitors on the Course of COVID19. Journal of inflammation research 2021;14:287-98. doi: 10.2147/jir.s292303 [published Online First: 2021/02/13]

14. Luxenburger H, Sturm L, Biever P, et al. Treatment with proton pump inhibitors increases the risk of secondary infections and ARDS in hospitalized patients with COVID-19: coincidence or underestimated risk factor? J Intern Med 2020 doi: 10.1111/joim.13121

15. Taştemur Ş, Ataseven H. Is it possible to use Proton Pump Inhibitors in COVID-19 treatment and prophylaxis? Medical hypotheses 2020;143:110018. doi: 10.1016/j.mehy.2020.110018 [published Online First: 2020/07/18]

16. Rho Y, Cho DY, Son Y, et al. COVID-19 International Collaborative Research by the Health Insurance Review and Assessment Service Using Its Nationwide Real-world Data: Database, Outcomes, and Implications. J Prev Med Public Health 2021;54(1):8-16. doi: 10.3961/jpmph.20.616 [published Online First: 2021/02/24]

17. Suchard MA, Simpson SE, Zorych I, et al. Massive parallelization of serial inference algorithms for a complex generalized linear model. ACM Trans Model Comput Simul 2013;23(1) doi: 10.1145/2414416.2414791 [published Online First: 2013/01/01]

18. Tian Y, Schuemie MJ, Suchard MA. Evaluating large-scale propensity score performance through real-world and synthetic data experiments. International journal of epidemiology 2018;47(6):200514. doi: 10.1093/ije/dyy120 [published Online First: 2018/06/26]

19. Austin PC. An Introduction to Propensity Score Methods for Reducing the Effects of Confounding in Observational Studies. Multivariate Behav Res 2011;46(3):399-424. doi: 10.1080/00273171.2011.568786 [published Online First: 2011/08/06]

20. Lipsitch M, Tchetgen Tchetgen E, Cohen T. Negative controls: a tool for detecting confounding and bias in observational studies. Epidemiology (Cambridge, Mass) 2010;21(3):383-8. doi:

10.1097/EDE.0b013e3181d61 eeb [published Online First: 2010/03/26] 
21. Voss EA, Boyce RD, Ryan PB, et al. Accuracy of an automated knowledge base for identifying drug adverse reactions. Journal of biomedical informatics 2017;66:72-81. doi: 10.1016/j.jbi.2016.12.005 [published Online First: 2016/12/21]

22. Schuemie MJ, Ryan PB, DuMouchel W, et al. Interpreting observational studies: why empirical calibration is needed to correct p-values. Stat Med 2014;33(2):209-18. doi: 10.1002/sim.5925 [published Online First: 2013/08/01]

23. Charpiat B, Bleyzac N, Tod M. Proton Pump Inhibitors are Risk Factors for Viral Infections: Even for COVID-19? Clinical drug investigation 2020;40(10):897-99. doi: 10.1007/s40261-020-00963-x [published Online First: 2020/08/12]

\section{Figures}

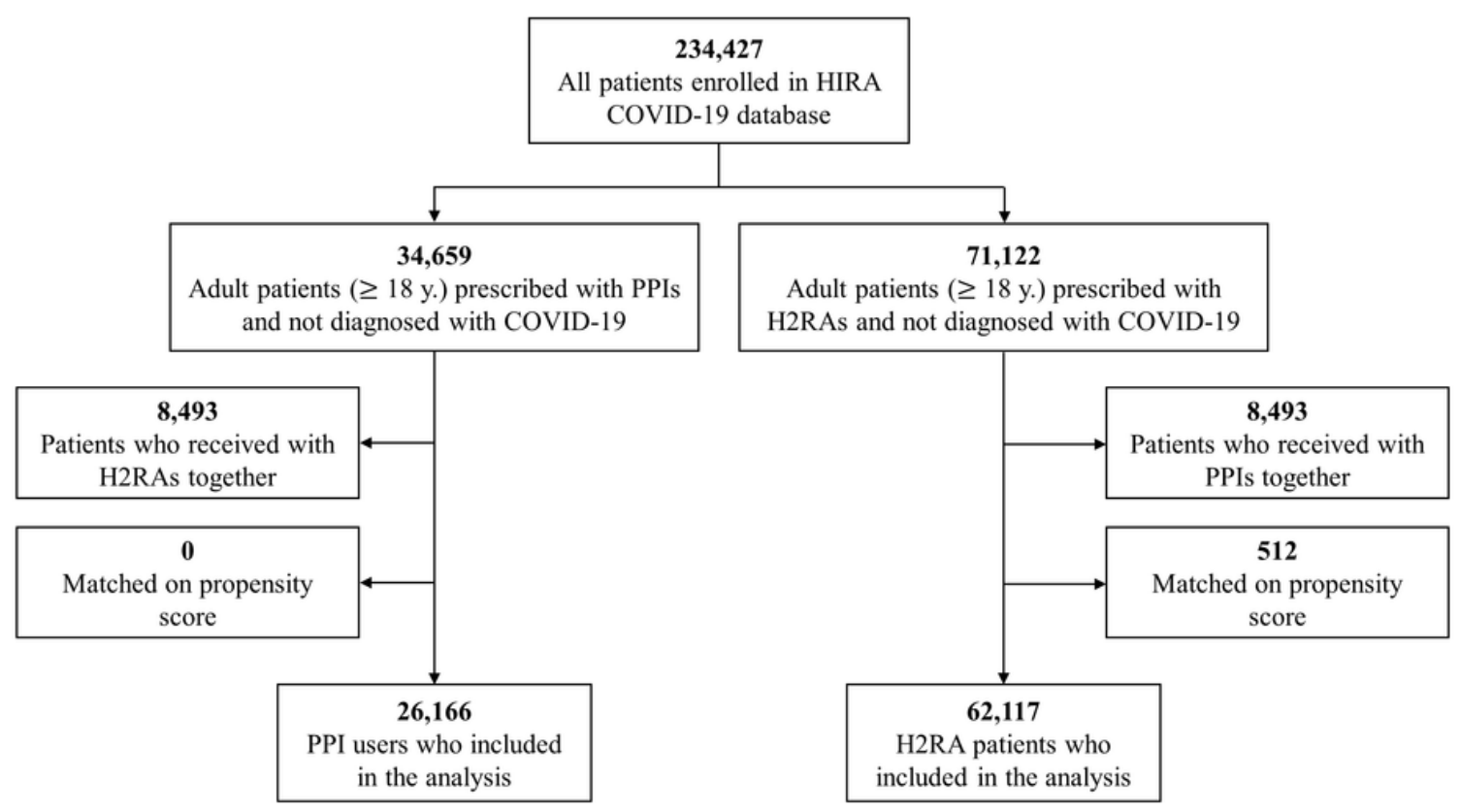

\section{Figure 1}

Flowchart of the patients who prescribed with acid-suppressants and not diagnosed with COVID-19.

\section{Supplementary Files}

This is a list of supplementary files associated with this preprint. Click to download. 